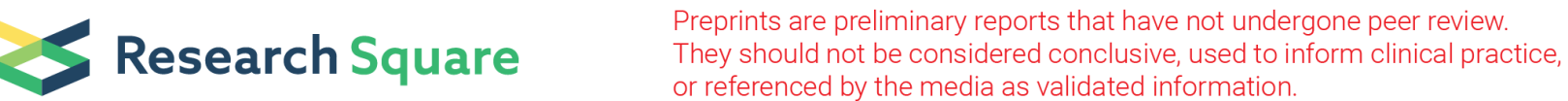

\section{Early Guideline-Directed Medical Therapy and In- Hospital Major Bleeding Risk in ST-Elevation Myocardial Infarction Patients Treated with Percutaneous Coronary Intervention: Findings from the CCC-ACS Project}

\section{Ziping Li}

Tianjin Medical University General Hospital

\section{Pengfei Yang}

Tianjin Medical University General Hospital

\section{A Geru}

Tianjin Medical University General Hospital

\section{Haonan Sun}

Tianjin Medical University General Hospital

\section{Hangkuan Liu}

Tianjin Medical University General Hospital

\section{Xiwen Song}

Tianjin Medical University General Hospital

\section{Zhengyang Jin}

Tianjin Medical University General Hospital

\section{Linjie Li}

Tianjin Medical University General Hospital

\section{Yongchen HaO}

Beijing Institute of Heart Lung and Blood Vessel Diseases: Capital Medical University Affiliated Anzhen Hospital

\section{Yongle Li}

Tianjin Medical University General Hospital

\section{Jing Liu}

Beijing Institute of Heart Lung and Blood Vessel Diseases: Capital Medical University Affiliated Anzhen Hospital

\section{Dong Zhao}

Beijing Institute of Heart Lung and Blood Vessel Diseases: Capital Medical University Affiliated Anzhen Hospital

Xin Zhou ( $\nabla$ xzhou@live.com ) 
Tianjin Medical University General Hospital https://orcid.org/0000-0003-1395-7103

\section{Qing Yang}

Tianjin Medical University General Hospital

\section{Research Article}

Keywords: Guideline-Directed Medical Therapy, ST-elevation myocardial infarction, Percutaneous coronary intervention, Bleeding

Posted Date: February 19th, 2021

DOl: https://doi.org/10.21203/rs.3.rs-216707/v1

License: (9) This work is licensed under a Creative Commons Attribution 4.0 International License. Read Full License 


\section{Abstract}

\section{BACKGROUND}

Previous reports demonstrated a bleeding avoidance potential of angiotensin converting enzyme inhibitor (ACEI)/angiotensin receptor blocker (ARB) and $\beta$-blocker. It remains unclear whether guideline-directed medical therapy [GDMT, i.e., the combined use of $\beta$-blocker, angiotensin converting enzyme inhibitor (ACEI)/angiotensin receptor blocker (ARB) and statin] confers protection against bleeding in the setting of high-intensity antithrombotic therapy.

\section{METHODS}

We assessed associations between the use of early (within the first 24 hours) GDMT and in-hospital major bleeds, ischemic events and mortality among ST-elevation myocardial infarction (STEMI) patients treated with percutaneous coronary intervention $(\mathrm{PCl})$ in the Improving Care for Cardiovascular Disease in China-Acute Coronary Syndrome project.

\section{RESULTS}

Among 34,538 STEMI patients without contra-indications to GDMT and eligible for analysis, 35.5\% received early GDMT. In a 1-to-2 propensity-score matched cohort, early GDMT was associated with a $28 \%$ reduction in major bleeds [odds ratio (OR): 0.72, 95\% confidence interval ( $\mathrm{Cl}$ ): 0.58 to 0.90], with parallel reductions in ischemic events (OR: $0.60,95 \% \mathrm{Cl}: 0.46$ to 0.78$)$ and in-hospital mortality (OR: 0.41, 95\% Cl: 0.30 to 0.57$)$. GDMT-associated reduction in major bleeds was consistently observed across different major bleeding definitions and in sensitivity analyses. Additionally, no significant interaction was observed in subgroup analyses.

\section{CONCLUSIONS}

In a large nationwide registry, early initiation of GDMT was associated with reduced risk for in-hospital major bleeds in STEMI patients treated with PCI. To improve the outcome of STEMI, further effort should be made to reinforce the use of GDMT in this patient population.

\section{What Is Known}

- Among stable and unstable coronary syndrome patients, STEMI patients had the highest risk of post$\mathrm{PCl}$ bleeding due to concomitant administration of high-intensity antithrombotic medications in short duration.

- ACEI/ARB and $\beta$-blocker, as the key component of GDMT, were demonstrated separately to have protective association against bleeding complications.

\section{What The Study Adds}


- Paralleled with reductions in ischemic events and in-hospital mortality, early initiation of GDMT (within the first 24 hours) was associated with a $28 \%$ reduction in major bleeding risk among STEMI patients treated with $\mathrm{PCl}$.

- To improve the outcome of STEMI, further effort should be made to reinforce the use of GDMT in this patient population.

\section{Introduction}

Attribute to continued improvement of technical and procedural advances, the outcomes of ST-elevation myocardial infarction (STEMI) patients after percutaneous coronary intervention (PCI) has been dramatically improved. ${ }^{1,2}$ Notably, there has been a concomitant increase in bleeding events associated with the use of more potent, longer-duration perioperative antithrombotic therapy in this patient population. ${ }^{3}$ In the SWEDEHEART registry, a gradually increased in-hospital bleeding from $0.5 \%$ in 1995 to a peak of $2 \%$ in 2005/2006 was observed, which was paralleled by an increasing use of $\mathrm{PCl}$ and antithrombotic therapy; notably, the implementation of bleeding avoidance strategy was associated with a concomitantly slight decline of bleeding rate to $1.3 \%$ in $2007-2010 .{ }^{4}$ Given that major bleeding is a costly complication associated with both short-term and long-term adverse outcomes following PCl, existing measures to reduce bleeding remain inadequate. ${ }^{5}$

Early initiation of guideline-directed medical therapy (GDMT), which includes a combined use of statin, $\beta$ blocker and angiotensin-converting enzyme inhibitor (ACEI)/angiotensin receptor blocker (ARB), has been recommended in current guidelines for the secondary prevention of atherosclerotic cardiovascular disease (ASCVD) after STEMI. ${ }^{6-8}$ Moreover, it's worth noting that recent studies have suggested that individual component of GDMT, such as ACEI/ARB and $\beta$-blocker, also had potential in reducing bleeding risk. $^{9,10}$ To our knowledge, the impact of GDMT on the risk of bleeding in eligible patients treated with PCI for STEMI remains unclear.

In the present study, we took advantage of the CCC-ACS project (Improving Care for Cardiovascular Disease in China-Acute Coronary Syndrome), an ongoing nationwide registry for acute coronary syndrome (ACS) in China, to examine the association between early (within the first 24 hours) GDMT use and major in-hospital bleeding risk among STEMI patients treated with $\mathrm{PCl}$ in contemporary practice.

\section{Methods}

\section{Study Design and Population}

The CCC-ACS project is an ongoing nationwide registry jointly initiated by the American Heart Association and the Chinese Society of Cardiology from 2014, aiming to improve the quality of care for ACS patients in China. Detailed information on the study design and methodology has been published previously. ${ }^{11}$ The CCC-ACS project was approved by the institutional review board of Beijing Anzhen Hospital, Capital 
Medical University, with a waiver for informed consent. This study is registered at the following URL: https://clinicaltrial.gov (unique identifier: NCT02306616).

In the present analysis, we included STEMI patients who underwent PCI during hospitalization and had no GDMT contraindications. GDMT was defined as the combination of the following three medications within 24 hours of STEMI onset: ACEI/ARB, $\beta$-blocker and statin. Non-GDMT was defined as at least one GDMT component was not used. Contraindications to statin include: active liver disease; persistent transaminase elevation of unknown cause; hypersensitivity to statin, myositis, myalgia, and rhabdomyolysis. Contraindications to $\beta$-blocker include: cardiogenic shock or unstable decompensated heart failure; sick-sinus syndrome (providing no permanent pacemaker), atrioventricular block of second and third degree; symptomatic bradycardia; hypotension and asthma. Contraindications to ACEI/ARB include: anuria renal failure with hyperkalemia, bilateral renal artery stenosis, isolated kidney with renal artery stenosis, pregnant and lactating women.

\section{Study Covariates}

The following variables were treated as covariates for multivariable adjustment and propensity score matching: demographics (age, gender), previous history (hypertension, diabetes, dyslipidemia, smoking, $\mathrm{MI}, \mathrm{PCl}$, coronary artery bypass grafting, heart failure, atrial fibrillation, renal failure, ischemic stroke, hemorrhagic stroke, chronic obstructive pulmonary disease, peripheral vascular disease), on-admission clinical characteristics [peak levels of creatine kinase MB (CKMB) isoform, Killip class, serum levels of low density lipoprotein cholesterol (LDL-C), high density lipoprotein cholesterol (HDL-C) and triglycerides (TG), levels of systolic and diastolic blood pressure (SBP and DBP), heart rate, estimated glomerular filtration rate (eGFR) and baseline hemoglobin), pre-hospital medications (pre-hospital thrombolysis, aspirin, $\mathrm{P}_{2} \mathrm{Y}_{12}$ inhibitors, statins, $\beta$-blockers, ACEls/ARBs, aldosterone antagonists and oral anticoagulants)], in-hospital medications [dual antiplatelet therapy (DAPT) status, aldosterone antagonists, oral anticoagulants, glycoprotein Ilb/llla inhibitors and perioperative anticoagulants (unfractionated heparin, low molecular weight heparin (LMWH) and others)] and PCl-related characteristics [time from symptom onset to admission, $\mathrm{PCl}$ types (primary $\mathrm{PCl}<12$ hours after symptom onset, primary $\mathrm{PCl} \geq 12$ hours after symptom onset, rescue $\mathrm{PCl}$ and elective $\mathrm{PCl}$ ) and radial route for $\mathrm{PCl}$ or not. eGFR was calculated according the equation by Chronic Kidney Disease Epidemiology Collaboration. ${ }^{12}$ The status of dual antiplatelet therapy (DAPT) within the first 24 hours was defined as the following four categories: non-DAPT (single antiplatelet therapy), non-loading DAPT (DAPT was not in loading dose), single-loading DAPT (one of DAPT in loading dose), and both-loading DAPT (DAPT both in loading dose). The loading dose of aspirin was defined as $\geq 150 \mathrm{mg}$. The loading dose of $\mathrm{P}_{2} \mathrm{Y}_{12}$ receptor inhibitor was defined as $\geq 300 \mathrm{mg}$ for clopidogrel and $\geq 180 \mathrm{mg}$ for ticagrelor. The definition of the above study variables is listed in Supplemental Table 1.

\section{Study Outcomes}


The CCC-ACS project routinely collected information concerning bleeding data as a part of in-hospital outcomes, which included: fatal bleeding, hemorrhagic stroke, bleeding in vital organs/locations (intracranial, spinal canal, retroperitoneal, pericardial, and intra-ocular with compromised vision), bleeding requiring clinical intervention (requiring pressors, surgery or intravenous vasoactive agents), hemoglobin drop related to bleed (the admission level minus the nadir level), and bleeding requiring blood transfusion and total amount of transfusion. Based on these information, we defined a composite of major bleeds using the following three major bleeding definitions: (1) Bleeding Academic Research Consortium (BARC) type $3 \mathrm{~b}-3 \mathrm{c}$ and type 5 , which is defined as a hemoglobin drop of $\geq 5 \mathrm{~g} / \mathrm{dL}$ or cardiac tamponade or bleeding requiring surgical intervention or bleeding requiring intravenous vasoactive agents (type 3b), intracranial hemorrhage (type 3c), or fatal bleeding (type 5), respectively; ${ }^{13}$ (2) Thrombolysis In Myocardial Infarction (TIMI) major bleeding, which is defined as intracranial hemorrhage or clinically overt bleeding associated with a hemoglobin drop of $\geq 5 \mathrm{~g} / \mathrm{dL}$, or fatal bleeding; ${ }^{14}$ (3) PLATelet inhibition and patient Outcomes (PLATO) life threatening bleeding, which is defined as fatal bleeding, intracranial bleeding, intraoperative bleeding with cardiac tamponade, severe hypotension, hypovolemic shock due to bleeding and requiring either vasopressor or surgery, a hemoglobin drop of $\geq 5 \mathrm{~g} / \mathrm{dL}$, or the need for transfusion $>4 \mathrm{U}$ of whole blood or packed red blood cells. ${ }^{15}$ Coronary artery bypass grafting related bleeding was excluded.

We also examined the association between GDMT status and ischemic events and all cause in-hospital mortality. Ischemic events were defined as the occurrence of re-infarction, in-stent thrombosis (angiographically confirmed) and ischemic stroke.

\section{Statistical Analysis}

Continuous data with normal distribution are presented as means and standard deviations.

Nonparametric continuous data are presented as medians with interquartile ranges and categorical data are presented as number and percentage. We used propensity score-matching to balance the differences in patient demographics, medical history and pre-admission and in-hospital management strategies between GDMT and non-GDMT patients. We developed a non-parsimonious multivariable logistic regression model to estimate a propensity score for GDMT status (yes/no) as the dependent variable. Between-group imbalances were considered to be ideal if the absolute standardized difference (ASD) for a given covariate was less than $10 \%{ }^{16}$ (Stata command "stddiff"). Then, a propensity score matching of a maximal ratio of 1-to-2, without replacement, with a caliper width of 0.02 was performed (Stata command "calipmatch"). The risk of in-hospital bleeding, ischemic events, and mortality in the matched groups was assessed using a logistic regression model on the matched pairs.

For variables with missing values, we imputed the missing data using the sequential regression multiple imputation method by IVEware (version 0.2; Survey Research Center, University of Michigan, Ann Arbor, $\mathrm{MI}$ ) as previously described. ${ }^{17}$ It should be noted that, for BMI, the imputation was not performed due to a high missing rate (>25\%). Therefore, BMI-related subgroup analysis and sensitivity analysis are not based on the full data set. 
We performed the following interaction tests and subgroup analyses based on matched population, including age ( $<65$ years and $\geq 65$ years), sex, BMI $\left(<28 \mathrm{~kg} / \mathrm{m}^{2}\right.$ and $\left.\geq 28 \mathrm{~kg} / \mathrm{m}^{2}\right)$, eGFR $(<60$ $\mathrm{mL} / \mathrm{min} / 1.73 \mathrm{~m}^{2}$ and $\geq 60 \mathrm{~mL} / \mathrm{min} / 1.73 \mathrm{~m}^{2}$ ), DAPT status (full loading or not) and Killip class (>Class I vs. Class I).

Finally, we performed the following sensitivity analyses based on the matching cohort of 1-to-2: (1) excluding patients receiving DAPT with both in loading dose; (2) excluding patients died within 48 hours of admission; (3) excluding patients with Killip Class IV; (4) excluding patients receiving unfractionated heparin; (5) excluding patients who had previous history of hemorrhagic stroke. Additionally, a propensity score-matched with a maximal matching ratio of 1-to-3, and inverse probability weighting based on multivariate logistic regression (Stata command "teffects ipw") were used as sensitivity analysis to validate the primary findings. We used Stata version 15.1 (StataCorp, College Station, TX) for analysis. A two-tailed $P<0.05$ was considered statistically significant.

\section{Results}

\section{Patient characteristics}

From November 2014 to January 2019, 104,516 ACS patients were enrolled in the CCC-ACS project. As shown in Fig. 1, a total of 34,538 patients were included in the final analytic sample after excluding those admitted with a diagnosis of non-ST-elevation myocardial infarction, those who was not treated with PCl, those with missing value for time from symptom-onset to admission, and those with contraindications to GDMT components. Among them, there were 12,262 GDMT patients and 22,276 non-GDMT patients. As shown in Supplemental Table 2, compared with non-GDMT patients, GDMT patients were more likely to have high levels of admission levels of blood pressure and heart rate, lower levels of admission peak CKMB and lower Killip class. Moreover, GDMT patients were more likely to receive LMWH and aldosterone antagonist. After propensity-score matching, a cohort composed of 9,040 GDMT patients $(73.7 \%$ of the total GDMT population) and 18,038 non-GDMT patients was constructed, with well-balanced demographics, pre-admission characteristics, medical history, admission characteristics and in-hospital management strategies. The baseline characteristics of post-matching cohorts are shown in Table 1, and the ASD between pre- and post-matched cohorts are shown in Fig. 2. 
Table 1

Baseline characteristics between GDMT patients and non-GDMT patients in post-matched cohorts

\section{Characteristics}

No. of Patients $\mathrm{n}=$

27078
GDMT $n=$ 9040
Non-GDMT $n=$ 18038
ASD, $\%$

\section{Demographics}

Age, year

$60.7 \pm 12.2$

$60.6 \pm 12.2$

$60.7 \pm 12.2$

0.94

Male, n (\%)

21794 (80.5)

7272 (33.4)

14522 (66.6)

0.17

Medical history

Smoking, n (\%)

$13942(51.5)$

12978 (47.9)

Hypertension, $\mathrm{n}(\%)$

5233 (19.3)

Diabetes, $\mathrm{n}(\%)$

1485 (5.48)

Dyslipidemia, n (\%)

$\mathrm{Ml}, \mathrm{n}(\%)$

1221 (4.51)

$\mathrm{PCl}, \mathrm{n}(\%)$

1201 (4.44)

CABG, n (\%)

$38(0.14)$

COPD, n (\%)

$247(0.91)$

Heart failure, $n(\%)$

$121(0.45)$

Renal failure, $\mathrm{n}(\%)$

$148(0.55)$

Atrial fibrillation, $\mathrm{n}(\%)$

293 (1.08)

1577 (5.82)

177 (0.65)

$126(0.47)$

Peripheral vascular disease,

n (\%)

Clinical variables

SBP, $\mathrm{mmHg}$

$128 \pm 21.9$

$129 \pm 20.9$

$128 \pm 22.4$

6.81

$\mathrm{DBP}, \mathrm{mmHg}$

$78.7 \pm 13.7$

$79.3 \pm 13.7$

$78.5 \pm 14.2$

6.21

Heart rate, bpm

$78.2 \pm 15.6$

$78.7 \pm 13.9$

$78.0 \pm 16.3$

4.14

Killip class, n (\%)

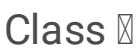

$20469(75.6)$

Class $\mathbb{Q}$

4861 (18.0)

889 (3.28)

4640 (33.3) $\quad 9302(66.7)$

0.48

4.13

0.42

0.35

0.37

0.25

0.31

0.27

0.60

0.32

0.13

0.25

0.80

0.95

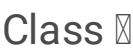

Page 8/21 


\begin{tabular}{|c|c|c|c|c|}
\hline Characteristics & $\begin{array}{l}\text { No. of Patients } n= \\
27078\end{array}$ & $\begin{array}{l}\text { GDMT } n= \\
9040\end{array}$ & $\begin{array}{l}\text { Non-GDMT } n= \\
18038\end{array}$ & $\begin{array}{l}\text { ASD, } \\
\%\end{array}$ \\
\hline 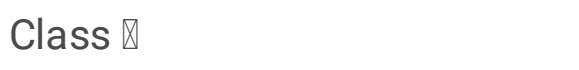 & $859(3.17)$ & $193(22.5)$ & $666(77.5)$ & \\
\hline CK-MB peak, $\mu \mathrm{g} / \mathrm{L}$ & $36.7(11.2-93.0)$ & $\begin{array}{l}34.5(11.8- \\
88.0)\end{array}$ & $36.8(10.9-96.2)$ & 2.14 \\
\hline LDL-C, mg/dL & $108(86.0-132)$ & $\begin{array}{l}108(86.0- \\
132)\end{array}$ & $108(85.0-132)$ & 0.67 \\
\hline $\mathrm{HDL}-\mathrm{C}, \mathrm{mg} / \mathrm{dL}$ & $41.0(34.0-49.0)$ & $\begin{array}{l}41.0(34.0- \\
48.0)\end{array}$ & $41.0(34.0-49.0)$ & 1.35 \\
\hline $\mathrm{TG}, \mathrm{mg} / \mathrm{dL}$ & $129(90.0-193)$ & $\begin{array}{l}131(92.0- \\
193)\end{array}$ & $127(89.0-193)$ & 0.19 \\
\hline eGFR, $\mathrm{mL} / \mathrm{min} / 1.73 \mathrm{~m}^{2}$ & $88.0 \pm 21.8$ & $88.3 \pm 21.2$ & $87.8 \pm 22.2$ & 2.11 \\
\hline $\begin{array}{l}\text { Hemoglobin on admission, } \\
\mathrm{g} / \mathrm{dL}\end{array}$ & $142(129-153)$ & $\begin{array}{l}142(129- \\
153)\end{array}$ & $141(129-153)$ & 1.43 \\
\hline \multicolumn{5}{|l|}{ Pre-hospital medications } \\
\hline $\begin{array}{l}\text { Pre-hospital thrombolysis, } \mathrm{n} \\
(\%)\end{array}$ & $429(1.58)$ & $142(33.1)$ & $287(66.9)$ & 0.16 \\
\hline Aspirin, n (\%) & $3889(14.4)$ & $1301(33.5)$ & 2588(66.5) & 0.13 \\
\hline P2Y12 inhibitor, n (\%) & $3047(11.3)$ & $1003(32.9)$ & $2044(67.1)$ & 0.75 \\
\hline Statin, n (\%) & $2480(9.16)$ & 799 (32.2) & $1681(67.8)$ & 1.67 \\
\hline Oral anticoagulants, n (\%) & $45(0.17)$ & $14(31.1)$ & $31(68.9)$ & 0.42 \\
\hline ß-blocker, n (\%) & $1143(4.22)$ & $406(35.5)$ & $737(64.5)$ & 2.00 \\
\hline ACEI/ARB, n (\%) & $1546(5.71)$ & $556(36.0)$ & $990(64.0)$ & 2.82 \\
\hline Aldosterone antagonist, n (\%) & $174(0.64)$ & $53(30.5)$ & $121(69.5)$ & 1.07 \\
\hline \multicolumn{5}{|l|}{ In-hospital medications } \\
\hline $\begin{array}{l}\text { DAPT status after admission, } \\
\mathrm{n}(\%)\end{array}$ & & & & 0.30 \\
\hline $\begin{array}{l}\text { DAPT was not in loading } \\
\text { dose }\end{array}$ & $1235(4.56)$ & $247(20.0)$ & $988(80.0)$ & \\
\hline One of DAPT in loading dose & $7953(29.4)$ & $2996(37.7)$ & $4957(62.3)$ & \\
\hline DAPT both in loading dose & $17890(66.1)$ & $5797(32.4)$ & $12093(67.6)$ & \\
\hline \multicolumn{5}{|l|}{ Anticoagulation therapy, $\mathrm{n}(\%)$} \\
\hline Unfractionated heparin & $1286(4.75)$ & $413(32.1)$ & $873(67.9)$ & 1.28 \\
\hline
\end{tabular}




\begin{tabular}{|c|c|c|c|c|}
\hline Characteristics & $\begin{array}{l}\text { No. of Patients } n= \\
27078\end{array}$ & $\begin{array}{l}\text { GDMT } n= \\
9040\end{array}$ & $\begin{array}{l}\text { Non-GDMT } n= \\
18038\end{array}$ & $\begin{array}{l}\text { ASD, } \\
\%\end{array}$ \\
\hline LMWH & $19447(71.8)$ & $6585(33.9)$ & $12862(66.1)$ & 3.43 \\
\hline Others & $818(3.02)$ & $283(34.6)$ & $535(65.4)$ & 0.96 \\
\hline Oral anticoagulants, $\mathrm{n}(\%)$ & $125(0.46)$ & $40(32.0)$ & $85(68.0)$ & 0.43 \\
\hline Aldosterone antagonist, $\mathrm{n}(\%)$ & $4440(16.4)$ & $1556(35.1)$ & $2884(65.0)$ & 3.29 \\
\hline $\begin{array}{l}\text { Glycoprotein Ilb/llla inhibitor, } \\
\text { n (\%) }\end{array}$ & 11706 (43.2) & $3913(33.4)$ & $7793(66.6)$ & 0.17 \\
\hline \multicolumn{5}{|l|}{$\mathrm{PCl}$ related } \\
\hline \multicolumn{4}{|c|}{ Time from symptom-onset to hospital admission, $\mathrm{n}(\%)$} & 1.29 \\
\hline$<6 \mathrm{~h}$ & $12234(45.2)$ & $6098(49.8)$ & $6136(50.2)$ & \\
\hline $6-12 \mathrm{~h}$ & $4296(15.9)$ & $2108(49.1)$ & $2188(50.9)$ & \\
\hline $12-24 \mathrm{~h}$ & $2313(8.54)$ & $1160(50.2)$ & $1153(49.8)$ & \\
\hline$>24 \mathrm{~h}$ & $4371(16.1)$ & $2241(51.3)$ & $2130(48.7)$ & \\
\hline Radial route for $\mathrm{PCl}, \mathrm{n}(\%)$ & $25571(94.4)$ & $8551(33.4)$ & $17020(66.6)$ & 1.03 \\
\hline PCl type, n (\%) & & & & 1.13 \\
\hline Primary, $<12 \mathrm{~h}$ & $18315(67.6)$ & $6102(33.3)$ & $12213(66.7)$ & \\
\hline Primary, $\geq 12 \mathrm{~h}$ & $2501(9.24)$ & $819(32.8)$ & $1682(67.3)$ & \\
\hline Rescue & $354(1.31)$ & $104(29.4)$ & $250(70.6)$ & \\
\hline Elective & $5908(21.8)$ & $2015(34.1)$ & $3893(65.9)$ & \\
\hline
\end{tabular}

Abbreviations: $A C E l$, angiotensin converting enzyme inhibitor; $A R B$, angiotensin receptor blocker; $C A B G$, coronary artery bypass grafting; CK-MB, creatine kinase MB isoform; COPD, chronic obstructive pulmonary disease; DAPT, dual antiplatelet therapy; DBP, diastolic blood pressure; eGFR, estimated glomerular filtration rate; GDMT, guideline-directed medical therapy; HDL-C, high density lipoprotein cholesterol; LDL-C, low density lipoprotein cholesterol; LMWH, low molecular weight heparin; MI, myocardial infarction; PCl, percutaneous coronary intervention; SBP, systolic blood pressure; TG, triglycerides.

\section{Associations between GDMT and major in-hospital bleeds, ischemic events and mortality}

In propensity score-matched cohort, a total of 404 composite major bleeds, 319 ischemic events, and 255 deaths were recorded, with incidence rate of $1.49 \%, 1.18 \%$, and $0.94 \%$, respectively. Compared with nonGDMT, GDMT was associated with a $28 \%$ reduction in major bleeds [odds ratio (OR) $0.72,95 \%$ confidence interval (Cl): 0.58-0.90], a $40 \%$ reduction in ischemic events (OR: $0.60,95 \% \mathrm{Cl}: 0.46-0.78$ ) and a $59 \%$ 
reduction in mortality (OR: $0.41,95 \% \mathrm{Cl}: 0.30-0.57)$. Notably, GDMT-associated reduction in bleeding risk was consistent for BARC- (OR: $0.77,95 \% \mathrm{Cl}$ : $0.62-0.97)$, TIMI- (OR: $0.66,95 \% \mathrm{Cl}: 0.50-0.87$ ), and PLATO(OR: 0.70, 95\%Cl: 0.55-0.89) defined major bleeds (Fig. 3). Moreover, as shown in Fig. 4, the reduction in major bleeding risk was generally consistent across subgroups, and no significant interaction was observed among subgroups.

\section{Sensitivity Analysis}

As shown in Fig. 5, the sensitivity analyses based on the 1-to-2 matched cohort revealed that the protective association of GDMT against major bleeds remained statistically significant after excluding patients receiving DAPT with both in loading dose, excluding patients died within 48 hours of admission, excluding patients with Killip Class IV, excluding patients receiving unfractionated heparin, and excluding patients with previous history of hemorrhagic stroke. Additional sensitivity analyses based on a 1-to-3 matched cohort (covariate balance after matching was shown in Supplemental Table 3 and Supplemental Fig. 1, and the results in detail was shown in Supplemental Fig. 2), revealed that GDMTassociated reduction in major bleeds was consistent for composite bleeds, as well as for BARC-, TIMI-, and PLATO-defined major bleeds. Moreover, GDMT was associated with similar magnitude of reductions in ischemic events (OR: $0.57,95 \% \mathrm{Cl}: 0.43-0.75$ ) and in-hospital mortality (OR: $0.39,95 \% \mathrm{Cl}: 0.28-0.56$ ) as observed in the 1-to-2 matched cohort. The results based on inverse probability weighting algorithm also confirmed the above findings (Supplemental Fig. 3).

\section{Discussion}

In a large nationwide registry in China, we showed that early initiation of GDMT, i.e., the combined use of statin, $\beta$-blocker and ACEI/ARB within the first 24 hours, was associated with a $28 \%$ reduction in major bleeding risk among STEMI patients treated with PCl. This finding was consistent by all methods used (propensity score matching and inverse probability weighting). Although the efficacy of GDMT in reducing in-hospital ischemic events and mortality in STEMI has been well documented, to our knowledge, our work for the first time demonstrated a protective association between early GDMT and bleeding risk in this patient population. Notably, in the present study, among patients treated with $\mathrm{PCl}$ and without a missing value for time delay for PCl, $0.5 \%$ (1824/36362, shown in Fig. 1) had a clear contraindication to GDMT, whereas only $35.5 \%(12,262 / 34,538)$ patients without a clear contra-induction received GDMT within the first 24 hours. This clearly indicates a large evidence-to-practice gap. Based on our findings, early GDMT should be further strengthened in STEMI patients, in terms of its potential benefit as a novel bleeding avoidance strategy, in addition to its proved efficacy in the secondary prevention of ASCVD.

Across different clinical presentations (chronic and acute coronary syndromes) indicated for $\mathrm{PCl}$, due to the concomitant administration of high-intensity antiplatelet and anticoagulant medications in short duration, STEMI patients had the highest risk of post-PCI bleeding, ${ }^{18}$ which would more likely to occur during hospitalization following $\mathrm{PCl},{ }^{19}$ and independently predicted mortality after STEMI. ${ }^{20}$ Current 
measures to reduce bleeding risk in this clinical scenario are still insufficient. Given the previous reports concerning the bleeding avoidance potential of the individual component of GDMT, especially $\beta$-blockers and ACEI/ARB, the potential of GDMT to reduce bleeding risk and its clinical value need to be reassessed. To the best of our knowledge, the present study is the first attempt to evaluate the association between GDMT and bleeding risk STEMI during hospitalization. Bleeding complications after PCl is generally categorized as procedure-related bleeding which usually occurs within 7 days and during hospitalization, and non-procedure related bleeding, also called spontaneous bleedings that occurs from 7 days after discharge. ${ }^{21}$ In this regard, our findings provide the first evidence that early GDMT was associated with reduced risk for procedure-related bleeding in STEMI patients.

Notably, recent evidence also supports a protective association between GDMT and non-procedure related bleeding following PCI. A post-hoc analysis of DAPT (Dual Antiplatelet Therapy) study evaluated the impact of optimal medical therapy (OMT; an equivalent term to GDMT) on cardiovascular outcomes in patients who underwent $\mathrm{PCl}$ with drug-eluting stent and completed 1 year of DAPT during an additional 18 months of continued use of clopidogrel or prasugrel. ${ }^{22}$ The patients included in the DAPT study were free of major adverse cardiovascular or cerebrovascular event, repeat revascularization or GUSTO (Global Use of Strategies to Open Occluded Arteries) moderate or severe bleeding at 12 months. During an additional 18 months of $\mathrm{P}_{2} \mathrm{Y}_{12}$ inhibition, 63\% patients were on OMT. After multivariable adjustment, the use of OMT was associated with a $30 \%$ reduction in GUSTO moderate or severe bleeding (hazard ratio, $0.70 ; 95 \%, 0.52-0.93$ ) during 12 to 30 months following PCl. Taken together, the findings from our study and the post-hoc analysis of DAPT study, clearly identified a GDMT-associated reduction in bleeding risk both during hospitalization and post-discharge. In our study, after excluding patients with clear contraindications to GDMT, the prescribing rate for GDMT within the first 24 hours was only $35.5 \%$. This percentage is significantly lower than reported by PROMETHEUS Registry $(69.4 \%) .{ }^{23}$ Given the clinical benefits of early GDMT presented in the present study, specific efforts should be made in the future to address the underuse of GDMT in clinical practice in China.

Due to the proved clinical efficacy in the secondary prevention of ASCVD, GDMT is currently recognized as the background treatment for STEMI patients. Therefore, it is difficult to establish a causal relationship between GDMT and its bleeding avoidance potential in randomized controlled trial. Currently, scatter evidence existed supporting the bleeding reduction potential for individual component of GDMT. For example, animal experiments have confirmed that ACEI/ARB is capable of maintaining gastric blood flow, inhibiting the inflammatory response caused by stress response, and protects the gastric mucosa from the impact of stress ulcer, thereby reducing gastrointestinal bleeding. ${ }^{24,25}$ Recent clinical observation also suggests that ACEIs/ARBs were associated with lower risk of major gastrointestinal bleeds in continuous-flow left ventricular assist device patients, which may be due to the prevention of arteriovenous malformations formation. ${ }^{10}$ With regards to $\beta$-blocker, accumulating evidence suggests that propranolol has a role in reducing the incidence of the first episode of upper gastrointestinal bleeding in patients with cirrhosis, with significant survival benefits. Therefore, non-selective $\beta$-blocker have been recommended for primary and secondary prevention of gastrointestinal bleeding in patients with cirrhosis 
and esophageal varices. ${ }^{9}$ Collectively, the available evidence of main components of GDMT, provides pharmacological proof that early GDMT would exert a synergistic effect on the reduced bleeding risk among STEMI patients treated with PCI.

Our study has the following limitations. First, as an observational study, we cannot establish a causal relationship between GDMT and major bleeding risk. Second, although we use propensity score matching to minimize bias, we cannot exclude the impact of unmeasured confounders. For example, the absence of information concerning cancer disease could be a limitation of this study. Cancers which diagnosed within the previous 12 months or ongoing active cancer treatment are associated with higher rates of inpatient bleeding. ${ }^{3}$ Third, considering clinically important differences in thrombogenicity and propensity for bleeding complications might exist between ethnic groups, ${ }^{26}$ our findings should not be overgeneralized. Future studies from other populations are warranted to confirm our findings.

In a large nationwide registry in China, among STEMI patients treated with $\mathrm{PCl}$, in parallel with reductions in ischemic events and in-hospital mortality, we demonstrated a protective association between early initiation of GDMT, i.e., the combined use of $\beta$-blocker, ACEI/ARB and statin within the first 24 hours, and reduced risk for in-hospital major bleeds. To improve the clinical care of STEMI in the Chinese population, further effort should be made to reinforce the use of GDMT in this patient population.

\section{Declarations}

\section{Acknowledgements}

The authors thank all hospitals participating in the CCC-ACS project (Improving Care for Cardiovascular Disease in China-Acute Coronary Syndrome; Supplemental Table 4 in Supplemental Material) for their invaluable contribution to this work.

\section{Contributors}

The article was written on behalf of the CCC-ACS project investigators. XZ and QY conceived and designed the study, supervised the analysis process, interpreted the data and revised the manuscript. ZL and PY analyzed the data and drafted the manuscript. ZL, PY, GA, HS, HL, XS, ZJ and LL helped to analyze the data and table and figure generation. YH, JL and DZ supervised the CCC-ACS project, and interpreted the data. All authors read and approved the final manuscript.

\section{Sources of Funding}

This work was supported by a collaborative program of the American Heart Association and the Chinese Society of Cardiology. The American Heart Association was funded by Pfizer and AstraZeneca for a quality improvement initiative through an independent grant for learning and change.

This work was also supported by Tianjin Municipal Science and Technology Commission (18ZXZNSY00290) and National Natural Science Foundation of China (81970304). 


\section{Data Availability}

The data, analytic methods, and study materials will be made available for onsite audit by third parties for purposes of reproducing the results or replicating the procedure.

\section{Disclosures}

None.

\section{References}

1. Puymirat E, Simon T, Cayla G, et al. Acute Myocardial Infarction: Changes in Patient Characteristics, Management, and 6-Month Outcomes Over a Period of 20 Years in the FAST-MI Program (French Registry of Acute ST-Elevation or Non-ST-Elevation Myocardial Infarction) 1995 to 2015. Circulation. 2017;136:1908-1919. doi: 10.1161/circulationaha.117.030798.

2. Maddox TM, Song Y, Allen J, et al. Trends in U.S. Ambulatory Cardiovascular Care 2013 to 2017: JACC Review Topic of the Week. J Am Coll Cardiol. 2020;75:93-112. doi: 10.1016/j.jacc.2019.11.011.

3. Urban P, Mehran R, Colleran R, et al. Defining High Bleeding Risk in Patients Undergoing Percutaneous Coronary Intervention. Circulation. 2019;140:240-261. doi:

10.1161/circulationaha.119.040167.

4. Simonsson $\mathrm{M}$, Wallentin $\mathrm{L}$, Alfredsson $\mathrm{J}$, et al. Temporal trends in bleeding events in acute myocardial infarction: insights from the SWEDEHEART registry. Eur Heart J. 2020;41:833-843. doi: 10.1093/eurheartj/ehz593.

5. Mcfadyen JD, Schaff M and Peter K. Current and future antiplatelet therapies: emphasis on preserving haemostasis. Nat Rev Cardiol. 2018;15:181-191. doi: 10.1038/nrcardio.2017.206.

6. Ibanez B, James S, Agewall S, et al. 2017 ESC Guidelines for the management of acute myocardial infarction in patients presenting with ST-segment elevation: The Task Force for the management of acute myocardial infarction in patients presenting with ST-segment elevation of the European Society of Cardiology (ESC). Eur Heart J. 2018;39:119-177. doi: 10.1093/eurheartj/ehx393.

7. O'gara PT, Kushner FG, Ascheim DD, et al. 2013 ACCF/AHA guideline for the management of STelevation myocardial infarction: a report of the American College of Cardiology Foundation/American Heart Association Task Force on Practice Guidelines. Circulation. 2013;127:e362-425. doi: 10.1161/CIR.0b013e3182742cf6.

8. [2019 Chinese Society of Cardiology (CSC) guidelines for the diagnosis and management of patients with ST-segment elevation myocardial infarction]. Zhonghua Xin Xue Guan Bing Za Zhi. 2019;47:766-783. doi: 10.3760/cma.j.issn.0253-3758.2019.10.003.

9. Ge PS and Runyon BA. The changing role of beta-blocker therapy in patients with cirrhosis. $J$ Hepatol. 2014;60:643-53. doi: 10.1016/j.jhep.2013.09.016.

10. Converse MP, Sobhanian M, Taber DJ, et al. Effect of Angiotensin II Inhibitors on Gastrointestinal Bleeding in Patients With Left Ventricular Assist Devices. J Am Coll Cardiol. 2019;73:1769-1778. doi: 
10.1016/j.jacc.2019.01.051.

11. Hao Y, Liu J, Liu J, et al. Rationale and design of the Improving Care for Cardiovascular Disease in China (CCC) project: A national effort to prompt quality enhancement for acute coronary syndrome. Am Heart J. 2016;179:107-15. doi: 10.1016/j.ahj.2016.06.005.

12. Levey AS, Stevens $\mathrm{LA}$, Schmid $\mathrm{CH}$, et al. A new equation to estimate glomerular filtration rate. Ann Intern Med. 2009;150:604-12. doi: 10.7326/0003-4819-150-9-200905050-00006.

13. Mehran R, Rao SV, Bhatt DL, et al. Standardized bleeding definitions for cardiovascular clinical trials: a consensus report from the Bleeding Academic Research Consortium. Circulation. 2011;123:273647. doi: 10.1161/circulationaha.110.009449.

14. Rao SV, O'grady K, Pieper KS, et al. A comparison of the clinical impact of bleeding measured by two different classifications among patients with acute coronary syndromes. J Am Coll Cardiol. 2006;47:809-16. doi: 10.1016/j.jacc.2005.09.060.

15. James S, Akerblom A, Cannon CP, et al. Comparison of ticagrelor, the first reversible oral P2Y(12) receptor antagonist, with clopidogrel in patients with acute coronary syndromes: Rationale, design, and baseline characteristics of the PLATelet inhibition and patient Outcomes (PLATO) trial. Am Heart J. 2009;157:599-605. doi: 10.1016/j.ahj.2009.01.003.

16. Mamdani M, Sykora K, Li P, et al. Reader's guide to critical appraisal of cohort studies: 2. Assessing potential for confounding. Bmj. 2005;330:960-2. doi: 10.1136/bmj.330.7497.960.

17. Hao Y, Liu J, Liu J, et al. Sex Differences in In-Hospital Management and Outcomes of Patients With Acute Coronary Syndrome. Circulation. 2019;139:1776-1785. doi:

10.1161/circulationaha.118.037655.

18. Loh JP, Pendyala LK, Torguson R, et al. Incidence and correlates of major bleeding after percutaneous coronary intervention across different clinical presentations. Am Heart J. 2014;168:248-55. doi: 10.1016/j.ahj.2014.05.018.

19. Giustino G, Mehran R, Dangas GD, et al. Characterization of the Average Daily Ischemic and Bleeding Risk After Primary PCI for STEMI. J Am Coll Cardiol. 2017;70:1846-1857. doi:

10.1016/j.jacc.2017.08.018.

20. Kikkert WJ, Van Geloven N, Van Der Laan MH, et al. The prognostic value of bleeding academic research consortium (BARC)-defined bleeding complications in ST-segment elevation myocardial infarction: a comparison with the TIMI (Thrombolysis In Myocardial Infarction), GUSTO (Global Utilization of Streptokinase and Tissue Plasminogen Activator for Occluded Coronary Arteries), and ISTH (International Society on Thrombosis and Haemostasis) bleeding classifications. J Am Coll Cardiol. 2014;63:1866-75. doi: 10.1016/j.jacc.2014.01.069.

21. Kazi DS, Leong TK, Chang TI, et al. Association of spontaneous bleeding and myocardial infarction with long-term mortality after percutaneous coronary intervention. J Am Coll Cardiol. 2015;65:141120. doi: 10.1016/j.jacc.2015.01.047.

22. Resor CD, Nathan A, Kereiakes DJ, et al. Impact of Optimal Medical Therapy in the Dual Antiplatelet Therapy Study. Circulation. 2016;134:989-998. doi: 10.1161/circulationaha.116.024531. 
23. Ge Z, Baber U, Claessen BE, et al. The prevalence, predictors and outcomes of guideline-directed medical therapy in patients with acute myocardial infarction undergoing $\mathrm{PCl}$, an analysis from the PROMETHEUS registry. Catheter Cardiovasc Interv. 2019;93:E112-e119. doi: 10.1002/ccd.27860.

24. Ender F, Labancz T and Rosivall L. Protective effects of the inhibition of the renin-angiotensin system against gastric mucosal lesions induced by cold-restraint in the rat. Acta Physiol Hung. 1993;81:13-8. doi.

25. Bregonzio $\mathrm{C}$, Armando I, Ando $\mathrm{H}$, et al. Angiotensin II AT1 receptor blockade prevents gastric ulcers during cold-restraint stress. Ann N Y Acad Sci. 2004;1018:351-5. doi: 10.1196/annals.1296.044.

26. Levine GN, Jeong YH, Goto $S$, et al. Expert consensus document: World Heart Federation expert consensus statement on antiplatelet therapy in East Asian patients with ACS or undergoing PCl. Nat Rev Cardiol. 2014;11:597-606. doi: 10.1038/nrcardio.2014.104.

\section{Figures}




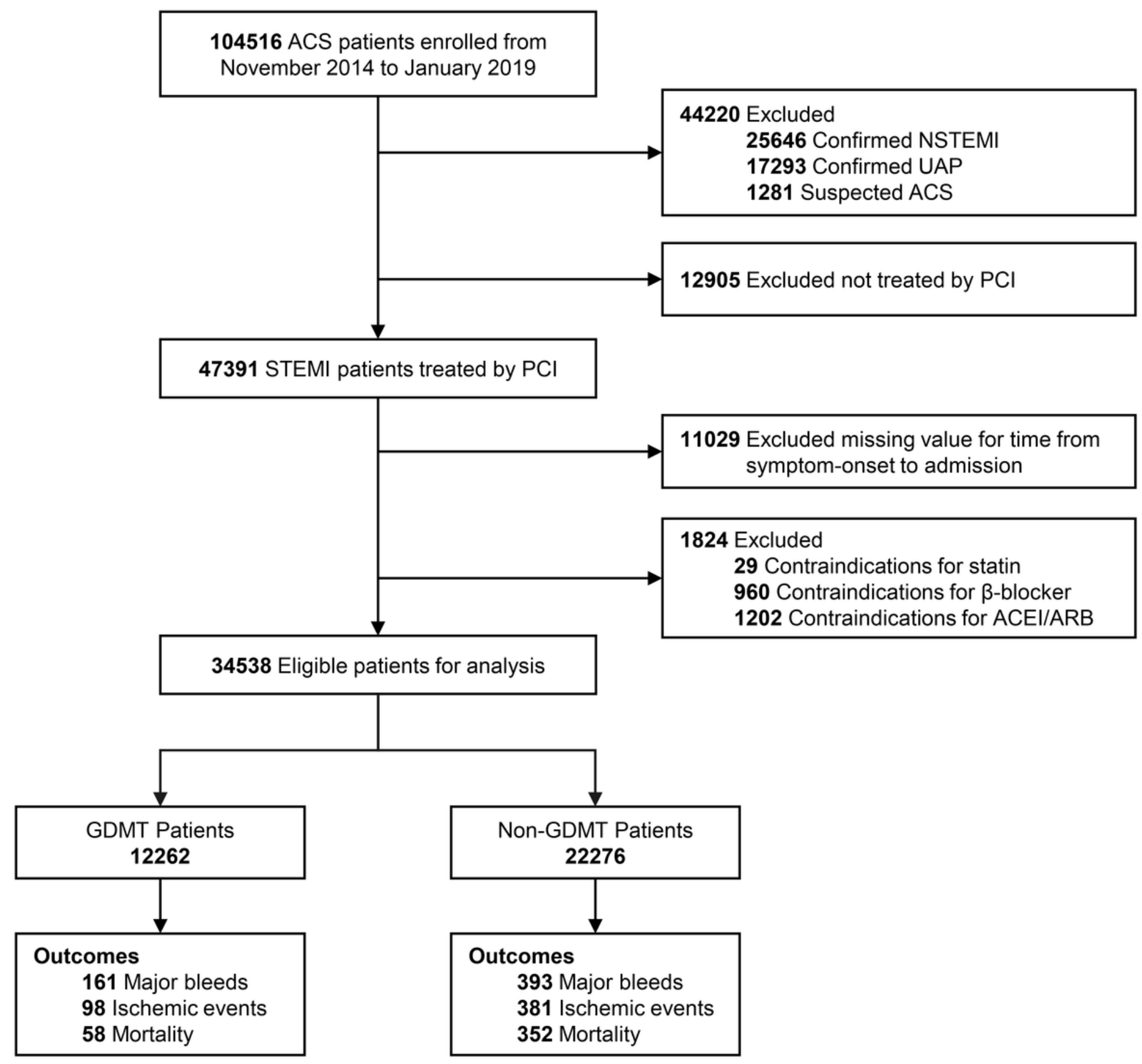

Figure 1

A schematic overview illustrating participant enrollment and the exclusion and inclusion criteria Abbreviations: ACEI, angiotensin-converting enzyme inhibitors; ACS, acute coronary syndrome; ARB, angiotensin receptor blockers; GDMT, guideline-directed medical therapy; NSTEMI, Non-ST-elevation Myocardial Infarction; PCl, percutaneous coronary intervention; STEMI, ST-elevation Myocardial Infarction; UAP, unstable angina pectoris. 


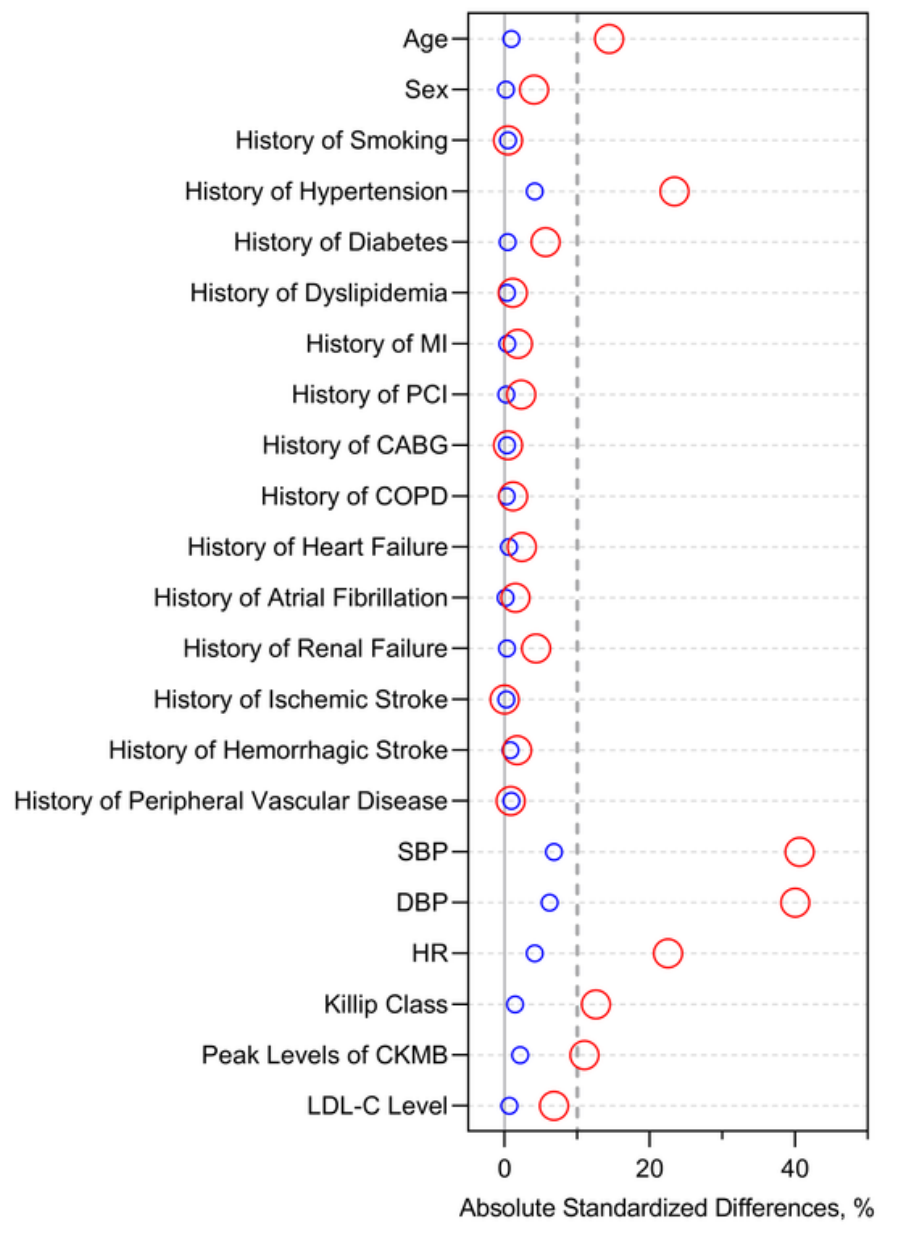

Pre-matching

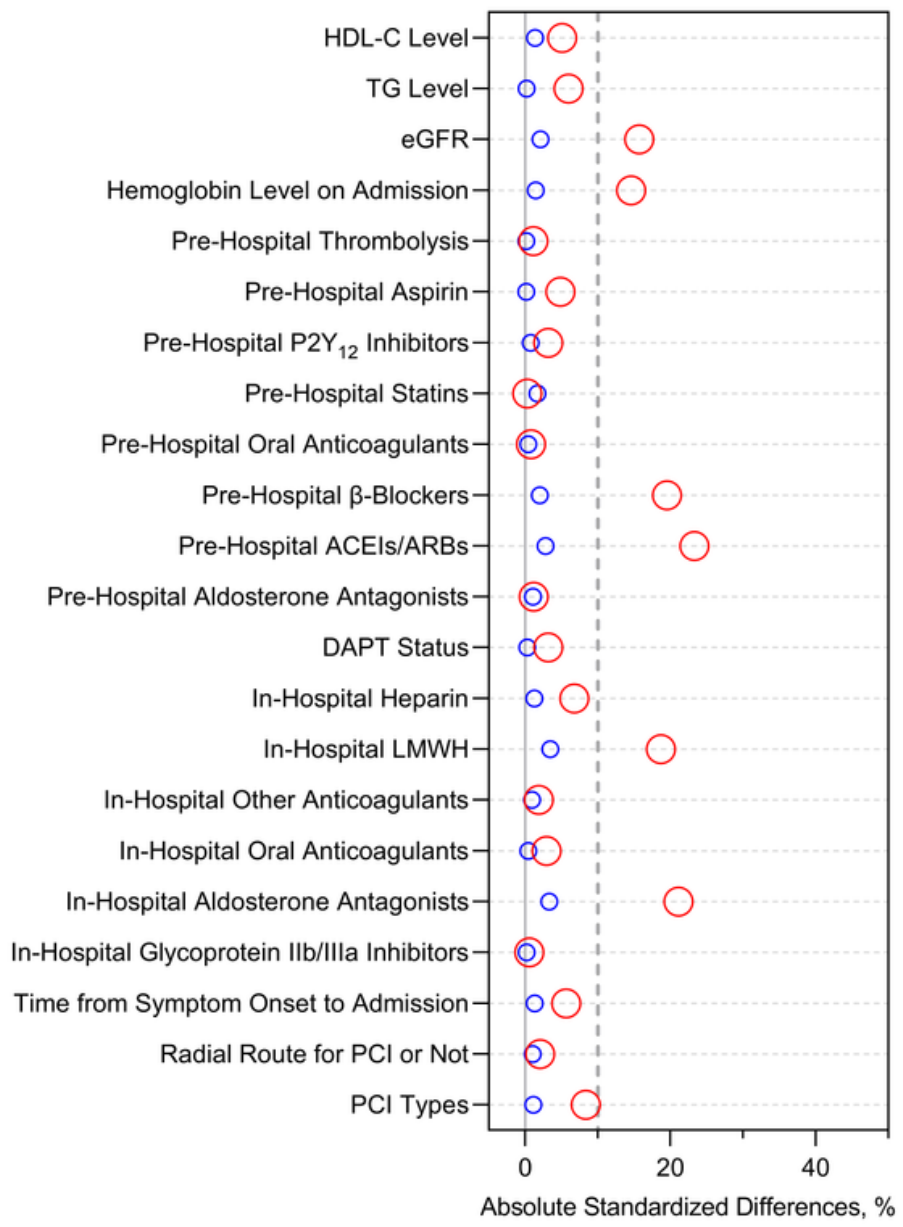

○ Post-matching

\section{Figure 2}

A Love-Plot illustrating the effect of matching were evaluated by comparing the absolute standardized difference Abbreviations: $A C E I$, angiotensin converting enzyme inhibitor; $A R B$, angiotensin receptor blocker; $C A B G$, coronary artery bypass grafting; $C K-M B$, creatine kinase $M B$ isoform; COPD, chronic obstructive pulmonary disease; DAPT, dual antiplatelet therapy; DBP, diastolic blood pressure; eGFR, estimated glomerular filtration rate; HDL-C, high density lipoprotein cholesterol; HR, heart rate; LDL-C, low density lipoprotein cholesterol; LMWH, low molecular weight heparin; $\mathrm{MI}$, myocardial infarction; $\mathrm{PCl}$, percutaneous coronary intervention; SBP, systolic blood pressure; TG, triglycerides. 


\begin{tabular}{|c|c|c|c|c|c|c|}
\hline & $\begin{array}{c}\text { Total } \\
\mathrm{n}=27078\end{array}$ & $\begin{array}{c}\text { GDMT } \\
\mathrm{n}=9040\end{array}$ & $\begin{array}{c}\text { Non-GDMT } \\
\mathrm{n}=18038\end{array}$ & \multicolumn{2}{|c|}{ Odd Ratio and 95\% Confidence Interval } & $P$ \\
\hline & & & & Favors GDMT & ivors Non-GDMT & \\
\hline Major bleeds & $404(1.49)$ & $108(1.19)$ & $296(1.64)$ & $\longrightarrow$ & $0.72(0.58$ to 0.90$)$ & 0.004 \\
\hline BARC type $3 b-3 c$ and type 5 & $364(1.34)$ & $102(1.13)$ & $262(1.45)$ & $\longrightarrow$ & $0.77(0.62$ to 0.97$)$ & 0.029 \\
\hline TIMI major & $276(1.02)$ & $69(0.76)$ & $207(1.15)$ & $\longrightarrow$ & $0.66(0.50$ to 0.87$)$ & 0.003 \\
\hline PLATO life threatening major bleeds & $364(1.34)$ & $95(1.05)$ & $269(1.49)$ & 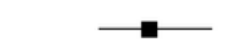 & $0.70(0.55$ to 0.89$)$ & 0.003 \\
\hline Ischemic events & $319(1.18)$ & $74(0.82)$ & $245(1.36)$ & $\longrightarrow$ & $0.60(0.46$ to 0.78$)$ & $<0.001$ \\
\hline Mortality & $255(0.94)$ & $44(0.49)$ & $211(1.17)$ & $\rightarrow-$ & $0.41(0.30$ to 0.57$)$ & $<0.001$ \\
\hline
\end{tabular}

\section{Figure 3}

The association between GDMT status and risk of in-hospital major bleeding, ischemia and mortality. Abbreviations: BARC, Bleeding Academic Research Consortium; GDMT, guideline-directed medical therapy; PLATO, PLATelet inhibition and patient Outcomes; TIMI, Thrombolysis in Myocardial Infarction. 


$\begin{array}{cccc}\text { Total } & \text { On GDMT } & \text { Off GDMT } & \text { Odds Ratios for Major Bleeding Risk and } \\ \mathrm{n}=27078 & \mathrm{n}=9040 & \mathrm{n}=18038 & 95 \% \text { Confidence Interval }\end{array}$

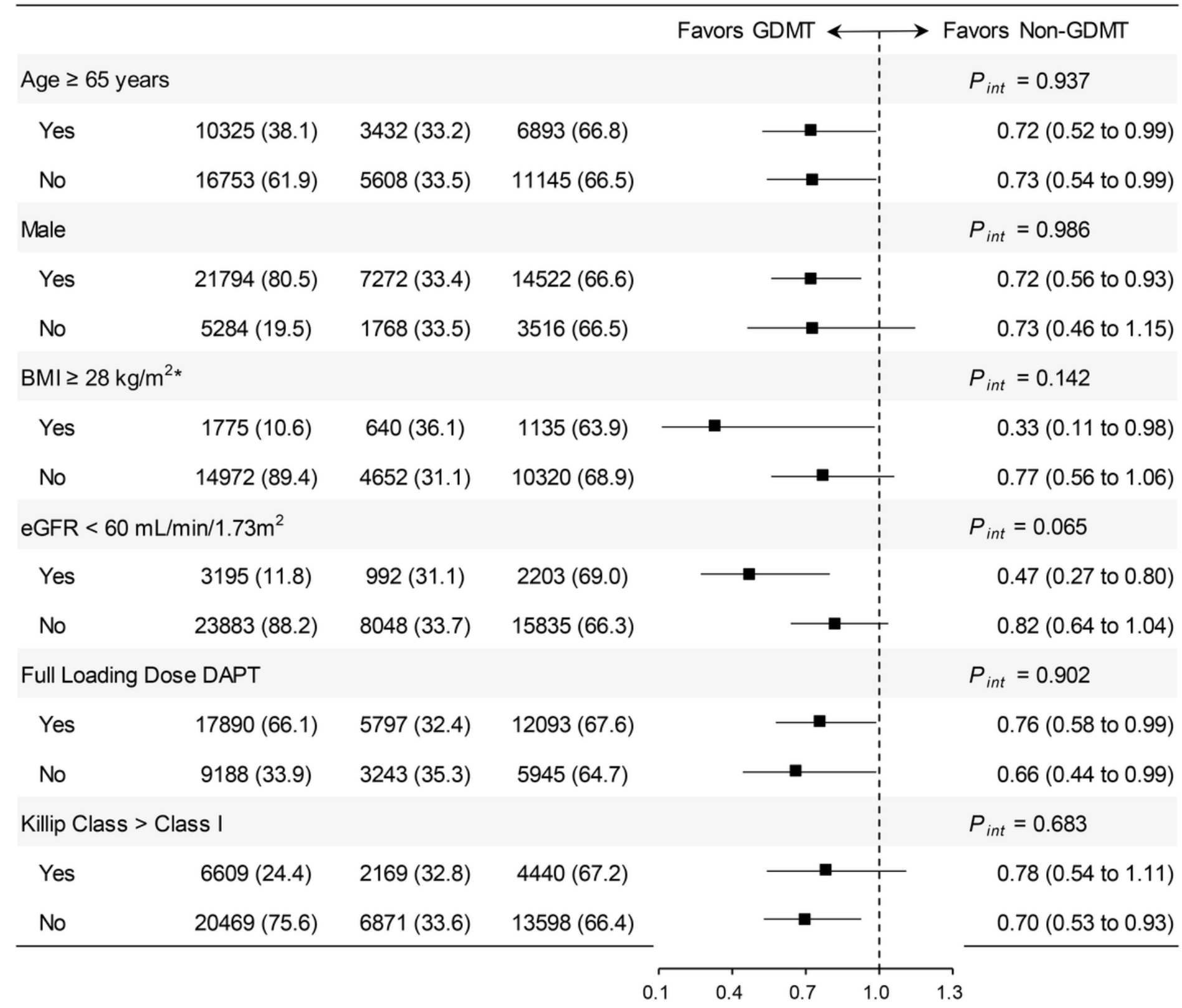

\section{Figure 4}

Subgroup analysis. ${ }^{*}$ BMI was derived from 16,747 participants without missing value. Abbreviations: BMI, Body Mass Index; DAPT, dual anti-platelet therapy; eGFR, estimated glomerular filtration rate; GDMT, guideline-directed medical therapy. 


\begin{tabular}{|c|c|c|c|c|c|}
\hline Total & GDMT & Non-GDMT & \multicolumn{2}{|c|}{$\begin{array}{l}\text { Odds Ratios for Major Bleeding Risk and } \\
95 \% \text { Confidence Interval }\end{array}$} & $P$ \\
\hline & & & \multicolumn{2}{|c|}{ Favors GDMT $\longleftrightarrow$ Favors Non-GDMT } & \\
\hline \multicolumn{6}{|l|}{ Total Effects (With 1:2 matched) } \\
\hline $404 / 27078(1.49)$ & $108 / 9040(1.19)$ & $296 / 18038(1.64)$ & $\longrightarrow$ & $0.72(0.58$ to 0.90$)$ & 0.004 \\
\hline \multicolumn{6}{|l|}{ Total Effects (With 1:3 matched) } \\
\hline $434 / 27789(1.56)$ & $83 / 6972(1.19)$ & $351 / 20817(1.69)$ & $\longrightarrow$ & $0.70(0.55$ to 0.89$)$ & 0.004 \\
\hline \multicolumn{6}{|c|}{ Total Effects (By inverse probability weighting) } \\
\hline $554 / 34538(1.56)$ & $161 / 12262(1.19)$ & $393 / 22276(1.69)$ & $\rightarrow$ & 0.73 (0.61 to 0.90$)$ & 0.002 \\
\hline \multicolumn{6}{|c|}{ Excluding Patients Receiving DAPT with Both In Loading Dose } \\
\hline $124 / 9188(1.35)$ & $33 / 3243(1.02)$ & $91 / 5945(1.53)$ & $\rightarrow$ & $0.66(0.44$ to 0.99$)$ & 0.043 \\
\hline \multicolumn{6}{|c|}{ Excluding Patients Died within 48 Hours of Admission } \\
\hline $396 / 26943(1.47)$ & $107 / 9024(1.19)$ & $289 / 17919(1.61)$ & $\longrightarrow$ & 0.73 (0.59 to 0.92$)$ & 0.006 \\
\hline \multicolumn{6}{|c|}{ Excluding Patients with Killip Class IV } \\
\hline $362 / 26219(1.38)$ & $104 / 8847(1.18)$ & $258 / 17372(1.49)$ & $\longrightarrow$ & 0.79 (0.63 to 0.99$)$ & 0.043 \\
\hline \multicolumn{6}{|c|}{ Excluding Patients Receiving Unfractionated Heparin } \\
\hline $367 / 25792(1.42)$ & $98 / 8627(1.14)$ & $269 / 17165(1.57)$ & $\longrightarrow$ & $0.72(0.57$ to 0.91$)$ & 0.006 \\
\hline \multicolumn{6}{|c|}{ Excluding Patients Who Had Previous History of Hemorrhagic Stroke } \\
\hline $345 / 26990(1.28)$ & $95 / 9020(1.05)$ & $250 / 17970(1.39)$ & $\longrightarrow$ & 0.75 (0.59 to 0.96$)$ & 0.020 \\
\hline & & 0.1 & 0.4 & 3 & \\
\hline
\end{tabular}

\section{Figure 5}

Sensitivity analysis. Abbreviations: DAPT, dual anti-platelet therapy; GDMT, guideline-directed medical therapy.

\section{Supplementary Files}

This is a list of supplementary files associated with this preprint. Click to download.

- SupplementalMaterial.docx 